\title{
The lactoferricin B-derived peptide, LfB17-34, induces melanogenesis in B16F10 cells
}

\author{
HSIU-CHIN HUANG ${ }^{1}$, HSUAN LIN ${ }^{1}$ and MIN-CHUAN HUANG ${ }^{2,3}$ \\ ${ }^{1}$ Renorigin Innovation Institute, Taipei 11560; ${ }^{2}$ Graduate Institute of Anatomy and Cell Biology, National Taiwan University \\ College of Medicine, Taipei 100; ${ }^{3}$ Research Center for Developmental Biology and Regenerative Medicine, \\ National Taiwan University, Taipei 10051, Taiwan, R.O.C.
}

Received July 2, 2016; Accepted January 30, 2017

DOI: $10.3892 / \mathrm{ijmm} .2017 .2884$

\begin{abstract}
Lactoferricin B (LfcinB), a peptide of bovine lactoferrin (LfB), exhibits multiple biological functions, including antimicrobial, antiviral, antioxidant and immunomodulatory activities. However, the role of LfcinB-related peptides in melanogenesis remains unclear. In this study, a set of five LfcinB-related peptides was examined. We found that LfB17-34, an 18-mer LfcinB-derived peptide, increased melanogenesis in B16F10 melanoma cells without significantly affecting cell viability. LfB17-34 increased in vitro tyrosinase activity and melanin content in a dose-dependent manner. The results of RT-qPCR and western blot analyses showed that LfB17-34 increased the mRNA and protein expression of tyrosinase and tyrosinase-related protein 1 (Trp1). Moreover, LfB17-34 inhibited the phosphorylation of MAPK/Erk, but not p38 and Akt, and constitutively active MEK was able to reverse the LfB17-34-enhanced pigmentation, melanin content, and tyrosinase activity, suggesting a role of Erk signaling in the process of LfB17-34-mediated pigmentation. Taken together, these results suggest that LfB17-34 induces melanogenesis in B16F10 cells primarily through increased tyrosinase expression and activity and that LfB17-34 could be further developed for the treatment of hypopigmentation disorders.
\end{abstract}

\section{Introduction}

Melanin is a natural pigment synthesized and stored in specialized organelles, termed melanosomes, in melanocytes (1). In the skin and hair, the melanosomes are transported to dendrites of melanocytes and then transferred to the neighboring keratinocytes. Insufficient synthesis of melanin leads to several human hypopigmentation disorders, such as hair graying and vitiligo (2). A recent study showed that $74 \%$ of people between 45 and 65 years of age have grey hair (3). Vitiligo is a

Correspondence to: Dr Min-Chuan Huang, Graduate Institute of Anatomy and Cell Biology, National Taiwan University College of Medicine, No. 1 section 1, Ren'ai Road, Taipei 100, Taiwan, R.O.C. E-mail: mchuang@ntu.edu.tw

Key words: melanin, lactoferrin, lactoferricin B, peptide, tyrosinase frequent cause of depigmentation worldwide with an estimated prevalence of $1 \%$ (4). Although there are several treatments for hypopigmentation disorders, the efficacy and safety remain unsatisfied and need to be improved (5). Therefore, it is urgent to develop novel agents for these diseases.

Melanogenesis is the process of melanin synthesis in melanocytes. Two types of melanin are present in mammals: a black/brown eumelanin and a red/yellow pheomelanin. The ratio of eumelanin and pheomelanin determines the diversity of skin and hair pigmentation in humans. Synthesis of both types of melanins begins with the amino acid L-tyrosine. Tyrosinase catalyzes L-tyrosine to L-3,4-dihydroxyphenyla lanine (L-DOPA), the rate-limiting step, for both eumelanin and pheomelanin. Eumelanin synthesis additionally requires the enzymes tyrosinase-related protein $2(\operatorname{Trp} 2)$ and $\operatorname{Trp} 1$, whereas pheomelanin additionally requires the amino acid cysteine (6). A well-known factor that can induce tyrosinase expression is $\alpha$-melanocyte stimulating hormone ( $\alpha-\mathrm{MSH})$, which binds the melanocortin 1 receptor (MC1R) to activate adenylyl cyclase to produce cAMP. The cAMP activates cAMP-dependent kinase A (PKA) and increases the expression of melanocyte-specific microphthalmia-associated transcription factor (Mitf), a master regulator for the expression of melanogenic enzymes tyrosinase, Trp1 and Trp2 (6). Identifying molecules that can modulate tyrosinase expression and activity is recognized to be crucial for developing agents to treat pigmentation disorders.

Lactoferrin (Lf) is a multifunctional glycoprotein in mammal milk which is known to exhibit a wide range of biological activities including antimicrobial, antiviral, antioxidant, and immunomodulatory activities. Lactoferricin B (LfcinB) is a 25-residue peptide (aa17-41) derived from pepsin-digested bovine lactoferrin (LfB).

LfcinB-related peptides share some activities with fulllength Lf, including antimicrobial, antiviral, antitumor, immunological, antihypertensive and anti-catabolic properties (7-11). LfB has been reported to inhibit pigmentation of melanoma cells (12). However, the effects of LfcinB-related peptides on melanogenesis remain unknown.

In this study, we found that three LfcinB-related peptides enhanced pigmentation in B16F10 melanoma cells. Among them, the 18-residue peptide LfB17-34, FKCRRWQWRM KKLGAPSI, strongly increased melanin synthesis, which is 
associated with elevated expression of melanogenic enzymes tyrosinase and Trp1. Moreover, LfB17-34 was able to decrease phosphorylation of Erk, a well-known signaling pathway to regulate melanogenesis. These findings suggest that LfB17-34 is a novel inducer of melanogenesis, whose stimulatory effect is at least partly through decreased phosphorylation of Erk and increased expression of tyrosinase and Trp1.

\section{Materials and methods}

Chemicals. Isobutylmethylxanthine (IBMX), L-DOPA, melanin, 3-(4,5-dimethylthiazol-2-yl)-2,5-diphenyltetrazolium bromide solution (MTT), paraformaldehyde, and all other chemicals were purchased from Sigma-Aldrich (St. Louis, MO, USA) unless otherwise indicated.

Preparation of peptides. All peptides were synthesized by MDBio, Inc. (Taipei, Taiwan) and the purity and composition of these peptides was confirmed by high performance liquid chromatography (HPLC) and mass spectrometry. Peptide stocks were prepared by dissolving $10 \mathrm{mg}$ of lyophilized peptide powder in $1 \mathrm{ml}$ of double deionized water $\left(\mathrm{ddH}_{2} \mathrm{O}\right)$, and then stored at $-20^{\circ} \mathrm{C}$.

Cell culture. B16F10 and B16F0 murine melanoma cells (American Type Culture Collection, Manassas, VA, USA) were cultured in phenol red-free Dulbecco's modified Eagle's medium (DMEM) (Thermo Scientific, Barrington, IL, USA) with $10 \%$ fetal bovine serum (FBS) (Gibco, Grand Island, NY, USA) and penicillin/streptomycin (100 IU/50 g/ml) in a humidified atmosphere containing $5 \% \mathrm{CO}_{2}$ at $37^{\circ} \mathrm{C}$. $\mathrm{B} 16 \mathrm{~F} 10$ cells $\left(2 \times 10^{5}\right)$ were transfected with $1 \mu \mathrm{g}$ of empty vector or vector coding constitutively active MEK (13) using Lipofectamine 2000 (Invitrogen Life Technologies, Carlsbad, CA, USA) for $48 \mathrm{~h}$ and then used for the assays.

Cell viability assay. Cells $\left(2 \times 10^{3}\right)$ were seeded in 96-well plates. After culture for different time periods, MTT was added to a final concentration of $0.5 \mathrm{mg} / \mathrm{ml}$ and incubated for $4 \mathrm{~h}$ at $37^{\circ} \mathrm{C}$ to allow MTT reduction. The formazan crystals were then dissolved in $10 \%$ sodium dodecyl sulfate (SDS) containing $0.01 \mathrm{~N} \mathrm{HCl}$ and absorbance was measured at the dual wavelengths of 570 and $630 \mathrm{~nm}$ with a spectrophotometer (VersaMax; Molecular Devices, Silicon Valley, CA, USA).

Melanin content assay. Melanin contents of cultured cells were measured according to the method (14) with a slight modification. Briefly, cells were seeded in a 6-well plate ( $2 \times 10^{4}$ cells/well) and incubated overnight to allow cells to adhere. After treating with various test samples for 4 or 5 days, cells were washed with phosphate-buffered saline (PBS), trypsinized, counted, and then lysed in $1 \mathrm{~N} \mathrm{NaOH}$ at $100^{\circ} \mathrm{C}$ for $1 \mathrm{~h}$. The melanin content in each sample was calculated by comparison of absorbance at $400 \mathrm{~nm}$ of unknown samples with a standard curve obtained using synthetic melanin. The results were converted to means $\pm \mathrm{SE}$ pg/cell from 3 independent experiments.

Tyrosinase activity assay. Tyrosinase enzyme activity of cultured cells were measured according to the a previous study (15) with a slight modification. Briefly, cells were seeded in a 6 -well plate $\left(2 \times 10^{4}\right.$ cells/well) and incubated for overnight. Cells were treated with peptides for 4 or 5 days. Cells were harvested and lysed with phosphate buffer ( $\mathrm{pH}$ 6.8) containing $1 \%$ Triton $\mathrm{X}-100$, followed by freezing and thawing. The supernatants were collected by centrifugation at $10,000 \mathrm{xg}$ for $10 \mathrm{~min}$ and the protein contents were determined using the BCA protein assay kit (Thermo Scientific, Rockford, IL, USA). For reactions, $100 \mu \mathrm{l}$ of cell lysates with the same amount of proteins were aliquoted into each wells of a 96-well plate, and then $100 \mu \mathrm{l}$ of $5 \mathrm{mM}$ L-DOPA were added to each wells. Following a $30-\mathrm{min}$ incubation period at $37^{\circ} \mathrm{C}$, the absorbance was measured spectrophotometrically at $475 \mathrm{~nm}$.

L-DOPA staining. L-DOPA staining was performed to measure tyrosinase enzyme activity in cultured cells. Cells were seeded in a 6 -well plate $\left(2 \times 10^{4}\right.$ cells/well $)$ and incubated overnight. After treating with various test samples in an incubator for 4 or 5 days, cells were washed with PBS and fixed with $2 \%$ paraformaldehyde for $20 \mathrm{~min}$. After washing with PBS for three times, the cells were incubated with $0.1 \% \mathrm{~L}-\mathrm{DOPA}$ in phosphate buffer $(\mathrm{pH} 6.8)$ at $37^{\circ} \mathrm{C}$ for $5 \mathrm{~h}$ and then photographed under a light microscope (16).

Reverse transcription-quantitative PCR(RT-qPCR). Total cellular RNA was isolated by using PureLink ${ }^{\mathrm{TM}}$ RNA mini kit (Invitrogen Life Technologies). For cDNA synthesis, $5 \mu \mathrm{g}$ of total RNA was used as templates in a $20 \mu 1$ reverse transcription reaction. The quantitative PCR system Mx3000P (Stratagene, La Jolla, CA, USA) was used for real-time PCR reactions. Reactions were performed in a $20-\mu 1$ volume with $1 \mu \mathrm{l} \mathrm{cDNA}$ and $10 \mu \mathrm{l}$ Brilliant ${ }^{\circledR} \mathrm{SYBR}^{\circledR}$-Green qPCR master mix (Stratagene). Samples were analyzed in triplicate, and product purity was checked by dissociation curves at the end of real-time PCR cycles. Relative quantity of gene expression normalized to $\beta$-actin was analyzed with MxPro software (Stratagene) (17). The following primer pairs were used: Mitf sense, 5'-AACAAGGGAACCATTCTCAAGG-3' and antisense, 5'-AGATCAGGCGAGCAGAGACC-3'; Tyr sense, 5'-CTCTGGGCTTAGCAGTAGGC-3' and antisense, 5'-GCAAGCTGTGGTAGTCGTCT-3'; Trp1 sense, 5'-GCTG CAGGAGCCTTCTTTCTC-3' and antisense, 5'-AAGACGCT GCACTGCTGGTCT-3'; Trp2 sense, 5'-GTCCTCCACTCTTT TACAGACG-3' and antisense, 5'-ATTCGGTTGTGACCAAT GGGT-3'; $\beta$-actin sense, 5'-AATCGTGCGTGACATCAAAG-3' and antisense, 5'-GAAAAGAGCCTCAGGGCAT-3'.

Western blot analysis. Protein lysates $(40 \mu \mathrm{g})$ were resolved by $8 \%$ SDS-PAGE, transferred to polyvinylidene difluoride (PVDF) membranes and then exposed to the appropriate antibodies. The primary antibodies used were p-Akt (\#4060), Akt (\#4691), p-Erk (\#9101), Erk (\#9102), p38 (\#9212), and p-p38 (\#9216) from Cell Signaling Technology (Danvers, MA, USA), $\beta$-actin from Sigma-Aldrich, and tyrosinase (H-109) (\#sc-15341), Trp1 (H-90) (\#sc-25543), Trp2 (H-150) (\#sc-25544), and Mitf (H-50) (\#sc-25386) antibodies were supplied by Santa Cruz Biotechnology, Inc. (Santa Cruz, CA, USA). The secondary antibodies used were horseradish peroxidase (HRP)-conjugated anti-mouse (115-035-003) or anti-rabbit (111-035-144) antibody (Jackson ImmunoResearch Laboratories, West Grove, PA, USA). 
A

LfcinB (aa17-41)

LfB17-34

LfB17-24

LfB20-29

LfB25-34

B

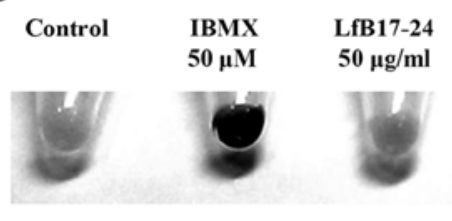

FKCRRWQWRMKKLGAPSITCVRRAF

FKCRRWQWRMKKLGAPSI

FKCRRWQW

RRWQWRMKKL

RMKKLGAPSI

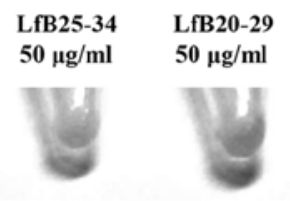

LfcinB

$50 \mu \mathrm{g} / \mathrm{ml}$

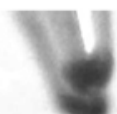

Figure 1. Lactoferricin B (LfcinB)-related peptides increase pigmentation in B16F10 cells. (A) Amino acid sequences of LfcinB-related peptides. (B) LfcinB-related peptides induced pigmentation of B16F10 melanoma cells. B16F10 cells were treated with $50 \mu \mathrm{g} / \mathrm{ml}$ peptides for 5 days or $50 \mu \mathrm{M}$ isobutylmethylxanthine (IBMX). Cells treated with solvent, $\mathrm{ddH}_{2} \mathrm{O}$, were used as controls. IBMX was used as positive control. Cells in 6-well plates were trypsinized and cell pellets were photographed. Representative images are shown from three independent experiments.

Protein signals were quantified by optical density ratios using $\beta$-actin expression as a control.

Statistical analyses. Quantitative data from at least three independent experiments are expressed as the means \pm standard deviation (SD). Student's t-tests were used to compare the differences between groups. $\mathrm{p}<0.05$ is considered statistically significant.

\section{Results}

LfcinB-related peptides increase pigmentation in B16F10 cells. To investigate whether LfcinB-related peptides affect pigmentation, we synthesized 5 LfcinB-related peptides. The amino acid sequences of these peptides are shown in Fig. 1A. The peptides and a chemical $50 \mu \mathrm{M}$ IBMX were used to treat mouse B16F10 melanoma cells for 5 days and their effects on pigmentation in cells were observed. IBMX, a non-specific inhibitor of cyclic AMP and cyclic GMP phosphodiesterases (PDEs), is a positive control that is commonly used compound to induce melanin synthesis. We found that three LfcinB-related peptides including LfB17-24, LfB17-34 and LfcinB, but not LfB25-34 and LfB20-29, were able to enhance pigmentation in B16F10 cells (Fig. 1B). Notably, LfB17-34 exhibited the most prominent effect on the enhancement of pigmentation.

LfB17-34 enhances melanin synthesis and secretion in a dosedependent manner without significant toxicity. To extend our preliminary finding on LfB17-34, we treated B16F10 cells with different concentrations of LfB17-34 ranging from 10 to $50 \mu \mathrm{g} / \mathrm{ml}$ and analyzed melanin contents in cells and conditioned medium. We found that LfB17-34 was able to enhance pigmentation of B16F10 cells in a dose-dependent manner (Fig. 2A). To further quantify the effect of LfB17-34 on pigmentation, we measured melanin content in both cells and conditioned medium. The results revealed that LfB17-34 significantly increased melanin content in both cells and conditioned medium in a dose-dependent manner (Fig. 2B). The concentration of LfB17-34 at $10 \mu \mathrm{g} / \mathrm{ml}$ was sufficient to significantly enhance melanin synthesis. To assess the toxicity of the LfB17-34 peptide, cell viability was analyzed by MTT assay. The results revealed that treatment of the B16F10 cells with $100 \mu \mathrm{g} / \mathrm{ml}$ of LfB17-34 for $48 \mathrm{~h}$ did not exert any significant effect on cell viability (Fig. 3). These results suggest that LfB17-34 enhances melanin synthesis and secretion in a dosedependent manner without significantly affecting cell viability.

LfB17-34 increases tyrosinase activity. Since tyrosinase has been demonstrated to be the most critical molecule to regulate melanin synthesis, we therefore analyzed whether LfB17-34 could increase tyrosinase enzyme activity by tyrosinase activity assay and L-DOPA staining. Consistent with our findings in the effect on melanin synthesis, LfB17-34 was able to enhance tyrosinase activity in a dose-dependent manner (Fig. 4A). L-DOPA staining of the cells treated with LfB17-34 also confirmed this effect (Fig. 4B). Our results suggest that LfB17-34 enhances tyrosinase activity in a dose-dependent manner.

LfB17-34 increases the expression of tyrosinase and Trp1. To investigate whether tyrosinase and other melanogenesis-related proteins were induced by LfB17-34, we performed western blot analysis and RT-qPCR. The results from western blot analysis revealed that LfB17-34 significantly increased the expression of tyrosinase and Trp1, but not that of Trp2 and Mitf (Fig. 5). We further assessed whether the mRNA levels of tyrosinase and Trp1 were also induced by LfB17-34. The results from RT-qPCR indicated that LfB17-34 significantly increased the mRNA levels of both tyrosinase and Trp1 (Fig. 6). These results suggest that LfB17-34 increases both the mRNA and protein levels of tyrosinase and Trp1.

LfB17-34 enhances melanogenesis in B16FO cells. To further confirm the effect of LfB17-34, we used another melanoma cell line, B16F0. We found that LfB17-34 was also able to increase 

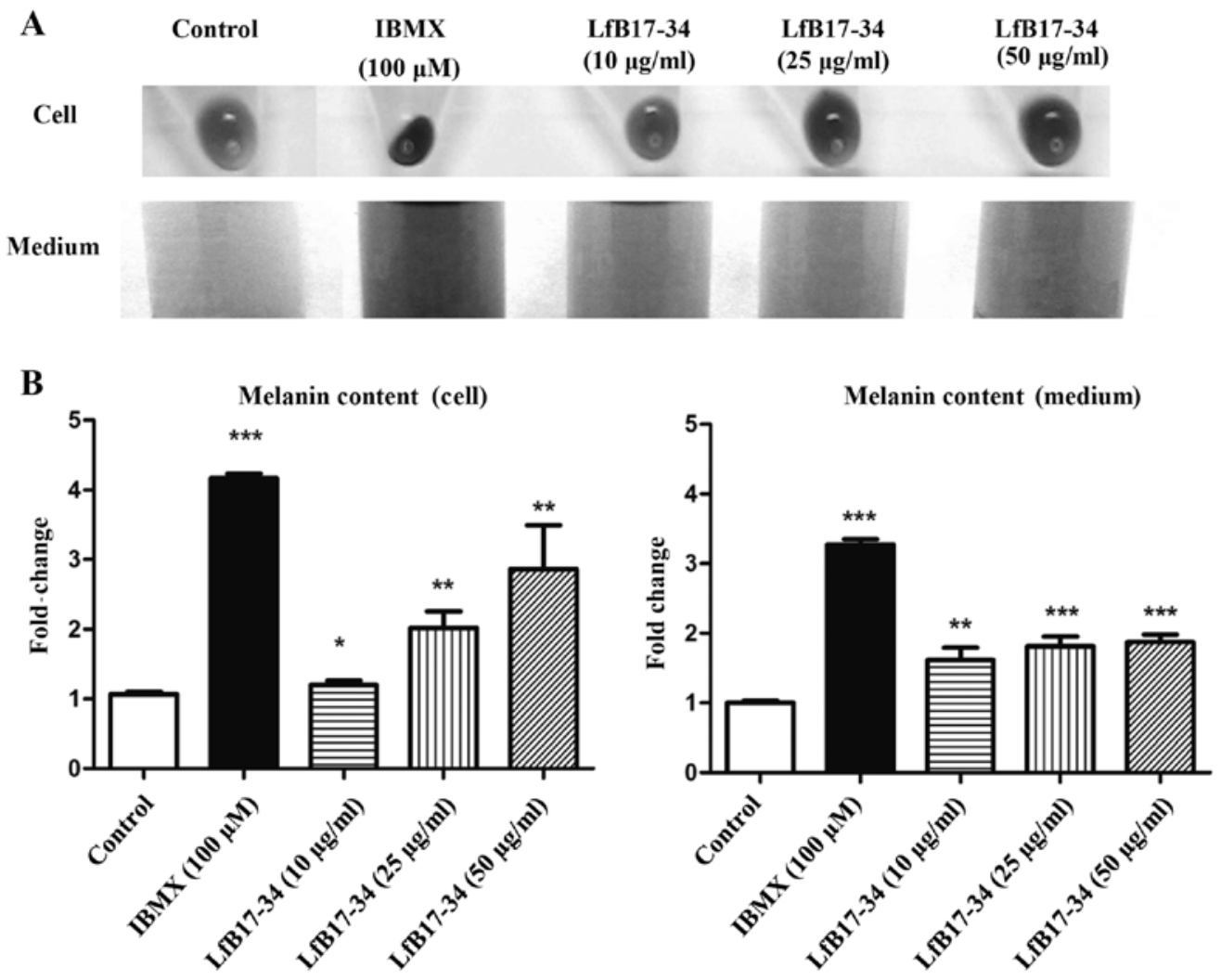

Figure 2. Bovine lactoferrin (LfB)17-34 enhances melanin synthesis and secretion in a dose-dependent manner. (A) LfB17-34 enhanced pigmentation in a dosedependent manner. B16F10 cells were cultured in 6-well plates and treated with isobutylmethylxanthine (IBMX) or LfB17-34 at different concentrations, as indicated, for 5 days. Cell pellets (cell) and conditioned medium (medium) were photographed. Representative images from three independent experiments are presented. (B) LfB17-34 enhanced melanin synthesis and secretion in a dose-dependent manner. Melanin content in B16F10 cells shown in (A) was measured. Data are represented as mean $\pm \mathrm{SD}$ from three independent experiments. $" \mathrm{p}<0.05,{ }^{* * *} \mathrm{p}<0.01$ and ${ }^{* * * *} \mathrm{p}<0.001$.

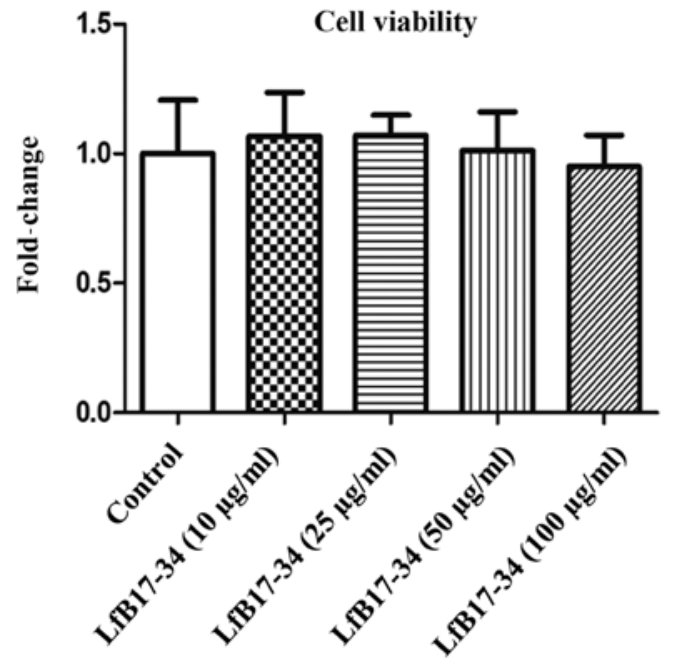

Figure 3. Bovine lactoferrin (LfB)17-34 does not significantly affect cell viability. B16F10 cells were treated with LfB17-34 at different concentrations as indicated for $48 \mathrm{~h}$. Cell viability was analyzed by MTT assay. Data are represented as the means $\pm \mathrm{SD}$ from three independent experiments.

cell pigmentation, melanin content, tyrosinase activity, and tyrosinase mRNA expression (Fig. 7). These data further confirm that LfB17-34 enhances melanogenesis.

LfB17-34 inhibits the phosphorylation of Erk. It is well known that the MAPK and Akt signaling pathways are involved in tyrosinase expression and melanogenesis. We therefore analyzed the phosphorylation of MAPK/Erk1/2, MAPK/ p38 and Akt by western blot analysis. Of note, we found that LfB17-34 was able to decrease the phosphorylation levels of Erk1/2, but not those of $\mathrm{p} 38$ and Akt (Fig. 8). It has been demonstrated that Erk1/2 signaling negatively regulates melanin synthesis in B16F10 cells $(19,20)$. To further determine whether p-Erk1/2 is involved in LfB17-34-induced melanogenesis, we transfected the B16F10 cells with an expression vector coding for constitutively active MEK. As expected, we found that constitutively active MEK reversed the LfB17-34-increased pigmentation, melanin content and tyrosinase activity (Fig. 9). Although the fold-changes in melanin content and tyrosinase activity varied in different experiments, the effects of LfB17-34 showed a similar tendency. Therefore, these findings suggest that the mechanism by which LfB17-34 enhances melanin synthesis may be mediated by the decrease in $\mathrm{p}-\mathrm{Erk} 1 / 2$ levels.

\section{Discussion}

Bovine Lf has been reported to exhibit a depigmenting effect on mouse melanoma cells (12). Unexpectedly, in this study, we found that LfB17-34, a bovine Lf-derived peptide, increased pigmentation in mouse melanoma cells. To the best of our knowledge, this is the first study showing that a Lf-derived peptide can stimulate pigmentation. We found that LfB17-34 significantly induced the expression of pigmentation-related genes, such as tyrosinase and Trp1. Moreover, LfB17-34 signif- 
A

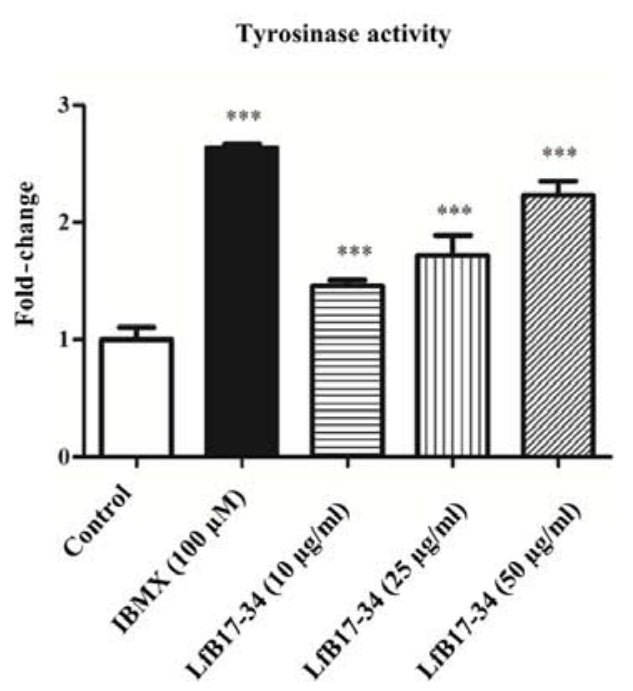

B

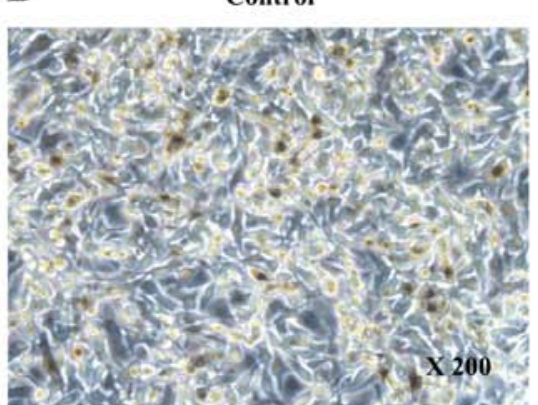

IBMX $100 \mu \mathrm{M}$

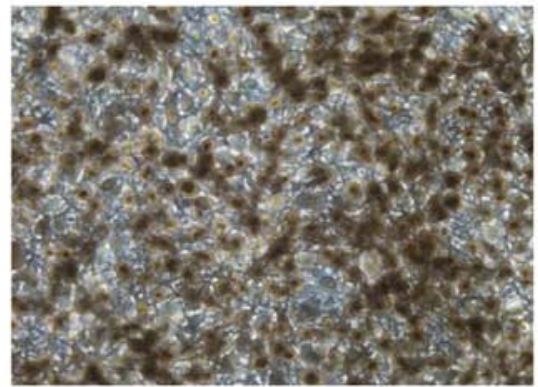

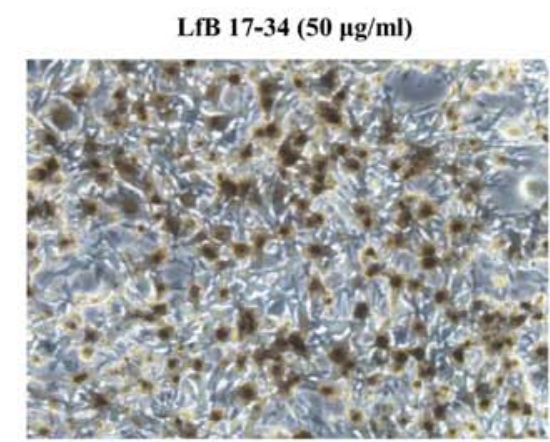

Figure 4. Bovine lactoferrin (LfB)17-34 increases tyrosinase activity. (A) LfB17-34 increased tyrosinase activity. B16F10 cells were cultured in 6-well plates and treated with isobutylmethylxanthine (IBMX) or LfB17-34 at different concentrations, as indicated, for 4-5 days. The cell lysates were used to perform tyrosinase activity assay. Data are represented as the means \pm SD from three independent experiments. ${ }^{* * *}$ p $<0.001$. (B) L-DOPA staining. B16F10 cells were cultured in 6 -well plates and treated with $100 \mu \mathrm{M}$ of IBMX or $50 \mu \mathrm{g} / \mathrm{ml}$ of LfB17-34 for 4-5 days. Control indicates cells treated with ddH $\mathrm{H}_{2} \mathrm{O}$. Then, the cells were used to perform L-DOPA staining and photographed. Representative images from three independent experiments are presented. 200 indicates 200 -fold magnification.
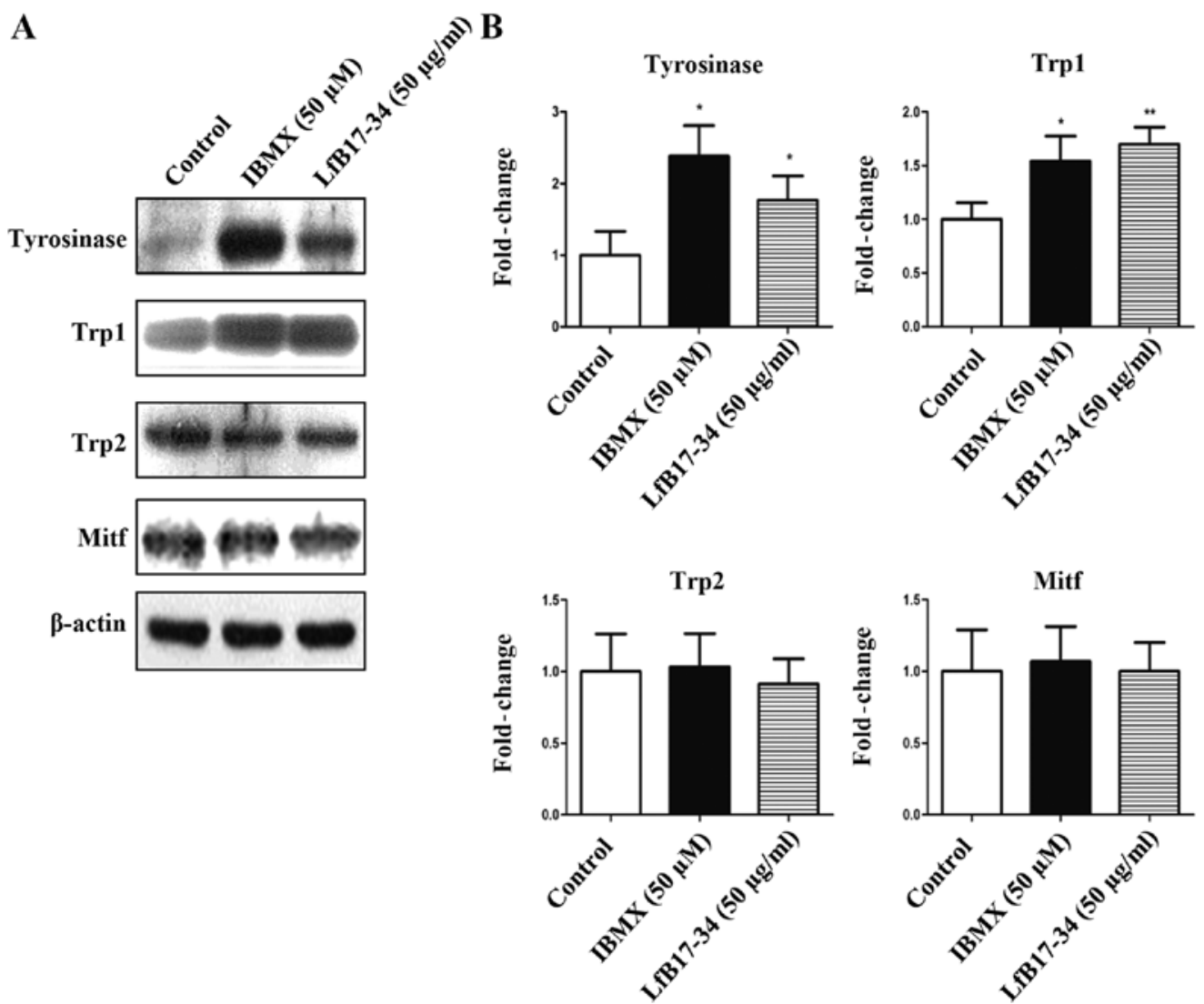

Figure 5. Bovine lactoferrin (LfB)17-34 increases the protein expression of tyrosinase and tyrosinase-related protein 1 (Trp1). (A) LfB17-34 increased tyrosinase and Trp1 expression. The expression of tyrosinase, Trp1, Trp2 and Mitf in B16F10 cells treated with $50 \mu \mathrm{M}$ of isobutylmethylxanthine (IBMX) or 50 $\mu \mathrm{g} / \mathrm{ml}$ of LfB17-34 was examined by western blot analysis. $\beta$-actin was used as internal control. Representative images from three independent experiments are presented. (B) Protein expression levels on western blot analyses were quantified by optical density ratios using $\beta$-actin expression as a control. Data are represented as the means \pm SD from three independent experiments. ${ }^{*} \mathrm{p}<0.05$ and ${ }^{* *} \mathrm{p}<0.01$ 

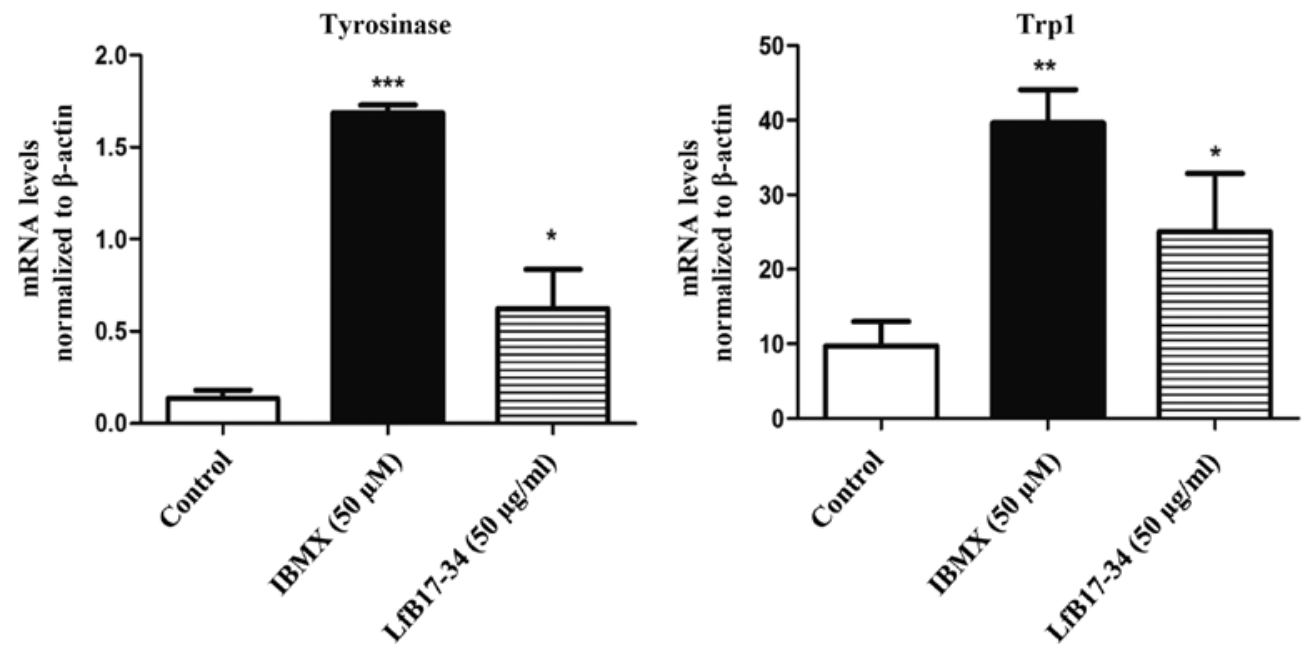

Figure 6. Bovine lactoferrin (LfB)17-34 increases the mRNA expression of tyrosinase and tyrosinase-related protein 1 (Trp1). The mRNA expression of tyrosinase and Trp1 in B16F10 cells treated with $50 \mu \mathrm{M}$ of isobutylmethylxanthine (IBMX) or 50 $\mu \mathrm{g} / \mathrm{ml}$ of LfB17-34 was analyzed by RT-qPCR. Data are represented as the means \pm SD from three independent experiments. ${ }^{*} p<0.05,{ }^{* *} p<0.01$ and ${ }^{* * * *} \mathrm{p}<0.001$.

\begin{tabular}{ccc} 
Control & $\begin{array}{c}\text { IBMX } \\
(100 \mu \mathrm{M})\end{array}$ & $\begin{array}{c}\text { LfB17-34 } \\
(50 \mu \mathrm{g} / \mathrm{ml})\end{array}$ \\
\hdashline & $\vdots$ & $\vdots$ \\
\hline & &
\end{tabular}

$\mathbf{B}$
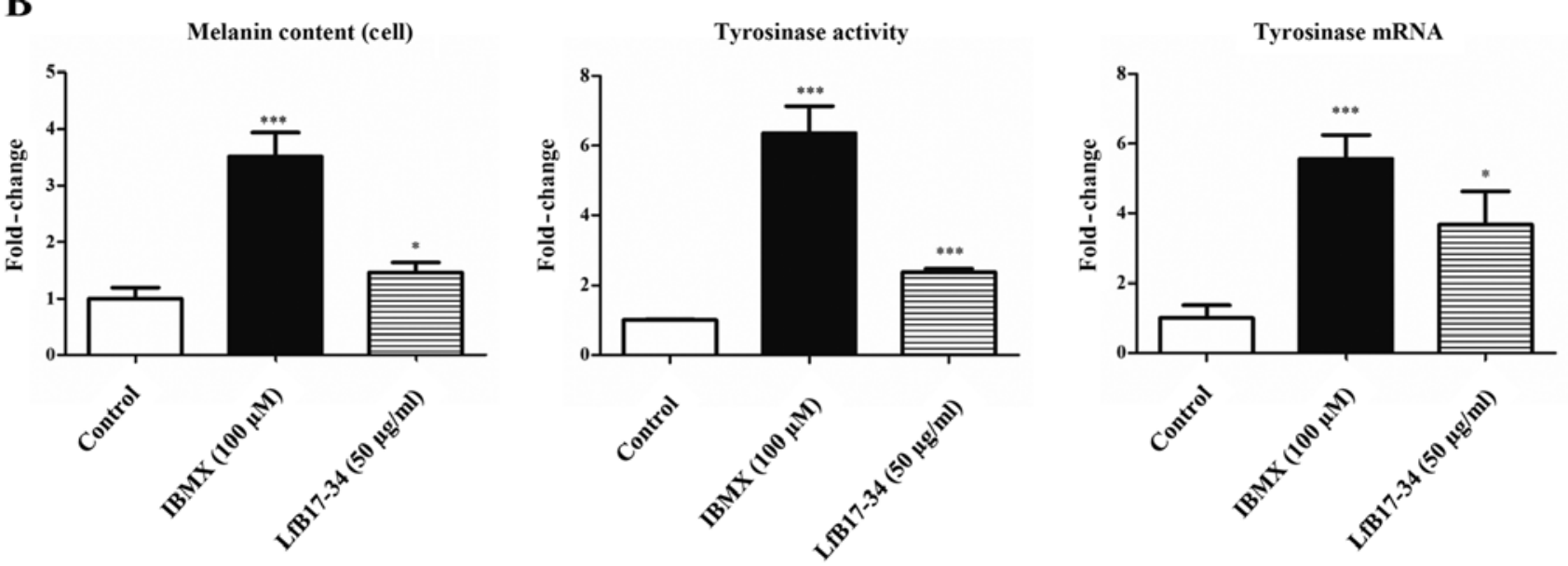

Figure 7. Bovine lactoferrin (LfB)17-34 increases melanogenesis in B16F0 cells. (A) LfB17-34 increased pigmentation in B16F0 cells. B16F0 cells were treated with $50 \mu \mathrm{g} / \mathrm{ml}$ of LfB17-34 peptide or $100 \mu \mathrm{M}$ of isobutylmethylxanthine (IBMX) for 5 days. Cells treated with $\mathrm{ddH}_{2} \mathrm{O}$ was used as control. IBMX treatment was a positive control. Cells in 6-well plates were then trypsinized and cell pellets were photographed. Representative images are shown from three independent experiments. (B) LfB17-34 increased melanin content, tyrosinase activity, and tyrosinase mRNA in B16F0 cells. Data are represented as the means \pm SD from three independent experiments. ${ }^{*} \mathrm{p}<0.05$ and ${ }^{* * *} \mathrm{p}<0.001$.

icantly inhibited Erk signaling, which negatively regulates melanogenesis. Therefore, we propose that LfB17-34 enhances pigmentation through increased expression of tyrosinase and Trp1, which is probably via decreased phospho-Erk.

Tyrosinase has been well demonstrated to be the crucial enzyme responsible for melanin synthesis. Hence, identifying molecules that can regulate tyrosinase expression and activity has been considered to be the key strategy to develop agents for treating pigmentation disorders. In this study, we found that LfB17-34 enhanced the expression of tyrosinase mRNA and protein as well as tyrosinase activity without significant cytotoxicity. Furthermore, the effect of LfB17-34 on tyrosi- nase activity and melanin content was dose-dependent. These results suggest that LfB17-34 induces pigmentation primarily through increased tyrosinase expression and activity and that LfB17-34 has great potential for drug development.

Mitf is regulated both transcriptionally and post-translationally (6). It is well known that cAMP activates PKA and in turn phosphorylates CREB, leading to activate the transcription of Mitf. However, in this study LfB17-34 did not significantly change phospho-CREB levels (data not shown). At the post-translational level, it has been reported that Mitf is phosphorylated at Ser73 by MAPK/Erk (18), which is known to facilitate ubiquitination and degradation of Mitf. Consistent 
A

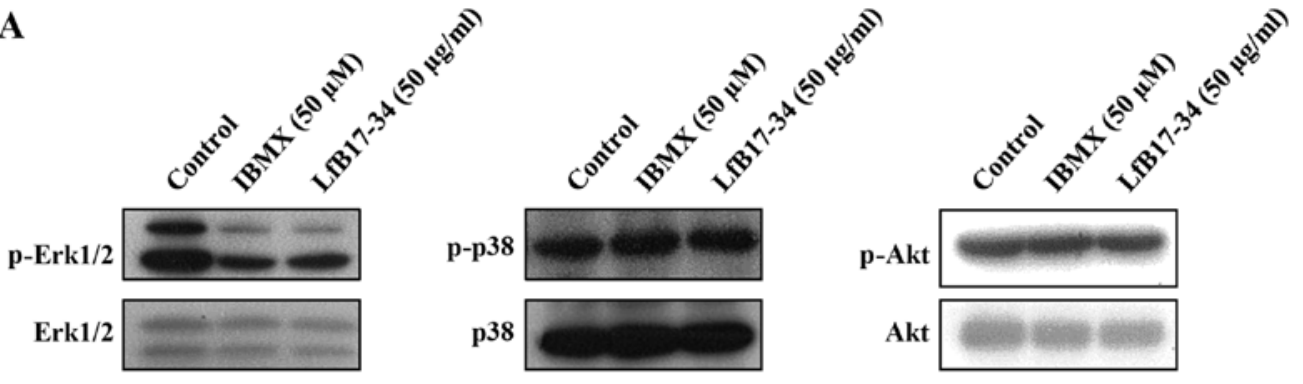

B
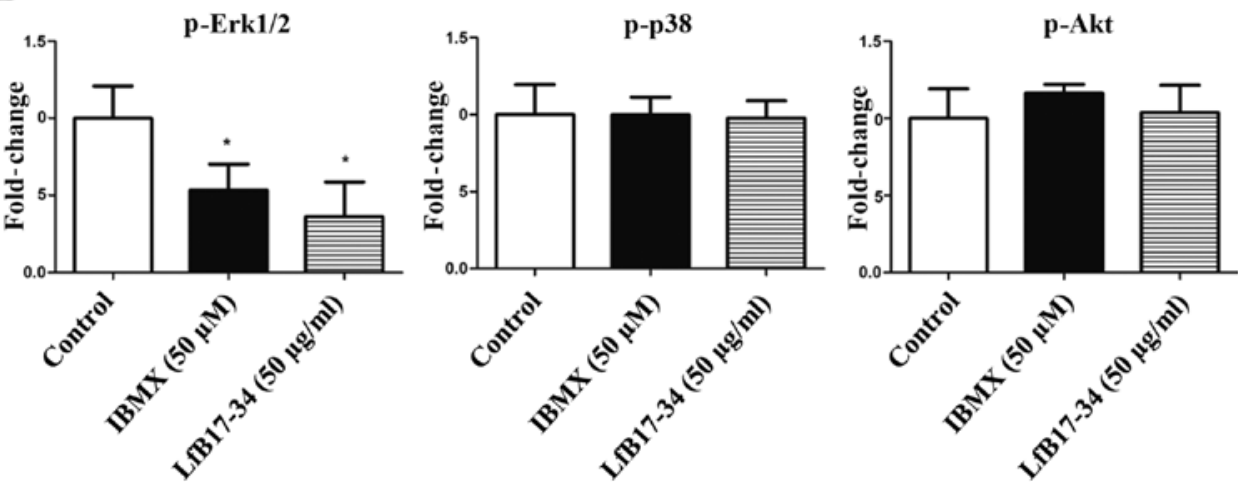

Figure 8. Bovine lactoferrin (LfB)17-34 inhibits the phosphorylation of Erk. (A) Phosphorylation of signaling proteins was examined by western blot analysis B16F10 cells were treated with $50 \mu \mathrm{M}$ of isobutylmethylxanthine (IBMX) or $50 \mu \mathrm{g} / \mathrm{ml}$ of LfB17-34. (B) Changes in protein phosphorylation. Signals of phosphoproteins on western blot analyses were quantified and normalized to their total protein levels. Data are represented as the means \pm SD from three independent experiments. ${ }^{*} \mathrm{p}<0.05$.

A

\begin{tabular}{|c|c|c|c|c|c|}
\hline \multicolumn{3}{|c|}{ Empty vector } & \multicolumn{3}{|c|}{ Constitutively active MEK } \\
\hline Control & $\begin{array}{c}\text { IBMX } \\
(100 \mu \mathrm{M})\end{array}$ & $\begin{array}{l}\text { LfB17-34 } \\
(50 \mu \mathrm{g} / \mathrm{ml})\end{array}$ & Control & $\begin{array}{c}\text { IBMX } \\
(100 \mu \mathrm{M})\end{array}$ & $\begin{array}{l}\text { LfB17-34 } \\
(50 \mu \mathrm{g} / \mathrm{ml})\end{array}$ \\
\hline & & & & & 6 \\
\hline
\end{tabular}

B

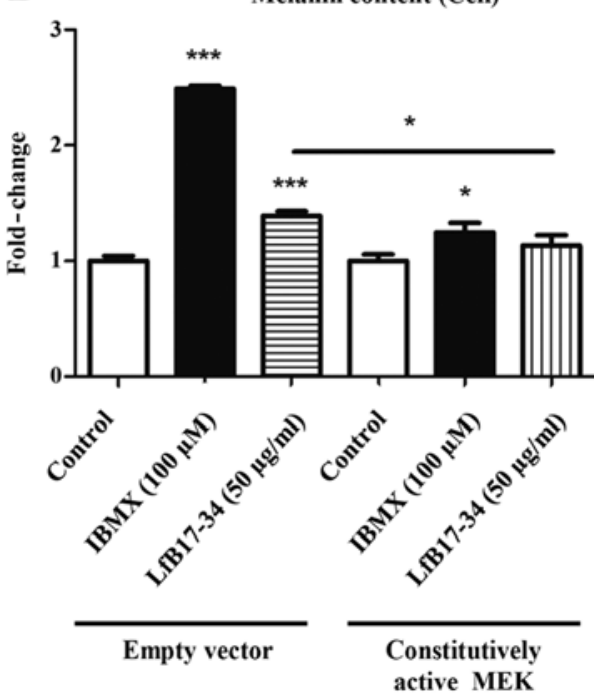

Tyrosinase activity

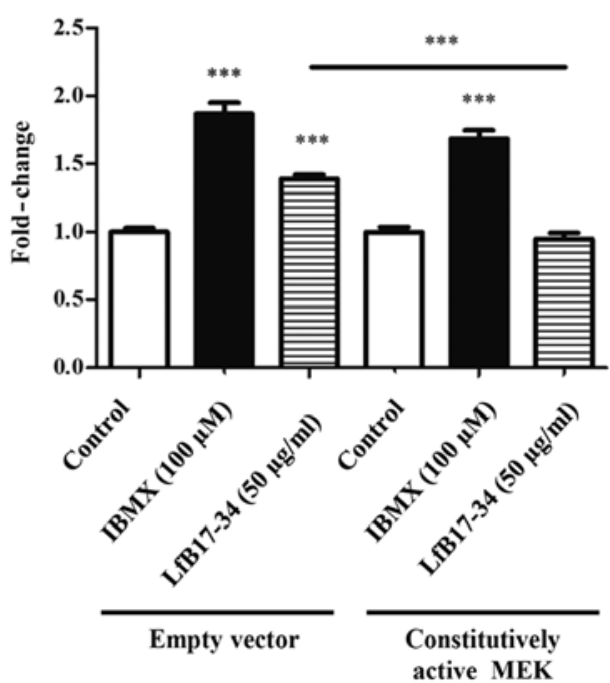

C

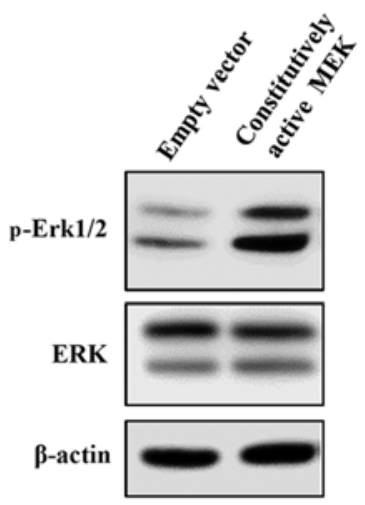

Figure 9. Constitutively active MEK decreases bovine lactoferrin (LfB)17-34-increased pigmentation, melanin content, and tyrosinase activity in B16F10 cells. (A) Constitutively active MEK decreased LfB17-34-increased pigmentation. B16F10 cells were transiently transfected with empty vector or vector coding for constitutively active MEK and then treated with $50 \mu \mathrm{M}$ of isobutylmethylxanthine (IBMX) or $50 \mu \mathrm{g} / \mathrm{ml}$ of LfB17-34. Cells treated with ddH $\mathrm{O}_{2} \mathrm{O}$ was used as controls. Representative images of cell pellets are shown from three independent experiments. (B) Constitutively active MEK decreased LfB17-34-increased melanin content and tyrosinase activity. (C) Western blot analyses showing that constitutively active MEK enhanced phospho (p)-Erk1/2 levels. $\beta$-actin was an internal control. Data are represented as the means \pm SD from three independent experiments. ${ }^{*} \mathrm{p}<0.05$ and ${ }^{* * * *} \mathrm{p}<0.001$. 
with this finding, activation of Erk has been reported to decrease Mitf expression and melanogenesis, whereas inhibition of Erk activity by PD98059 increases melanin production in B16F10 cells $(19,20)$. However, we consistently observed that LfB17-34 decreased phosphorylation of Erk but did not increase Mitf protein levels in B16F10 cells. In this case, LfB17-34 still could induce tyrosinase and Trp1 expression and enhance pigmentation. Similarly, we observed that $50 \mu \mathrm{M}$ IBMX increased pigmentation without elevated protein levels of tyrosinase and Trp1. There could be several explanations. Firstly, it is likely that LfB17-34 exerts its stimulatory effect on pigmentation by increasing the Mitf activity instead of the Mitf protein level via Erk signaling. Secondly, it is also possible that LfB17-34 induces melanin synthesis independent of Mitf. It is well known that tyrosinase, Trp1, and Trp2 are transcriptional targets of Mitf. However, LfB17-34 increased tyrosinase and Trp1 expression, but not Mitf and Trp2. Therefore, LfB17-34 may modulate other factors that can induce tyrosinase and Trp1 expression to enhance pigmentation. To understand the complicated process of melanogenesis, it will be of great interest to further investigate the detailed mechanism of LfB17-34-mediated pigmentation.

Among 5 LfcinB-related peptides analyzed, LfcinB (aa17-41), LfB17-24, and LfB17-34 exhibit stimulatory effect on pigmentation. LfB20-29 and LfB25-34 do not have any effects. These results suggest that the 8 amino acids (FKCRRWQW) in LfB17-24 could be essential for stimulating pigmentation. Moreover, we found that LfB17-34 exhibits stronger stimulatory effect than does LfcinB (aa17-41), suggesting that aa35-41 (TCVRRAF) could have inhibitory effect on pigmentation. Although the underlying mechanism remains to be further investigated, we speculate that LfB17-34 could form a specific conformation to interact with the surface molecules that can stimulate pigmentation signaling, such as elevated cAMP and/or decreased p-Erk.

LfcinB exhibits a diverse range of bioactivities. Therefore, LfcinB-related peptides are attractive targets for developing peptide drugs. In this study, and to the best of our knowledge, we are the first to report LfB17-34 as a novel stimulator of pigmentation. Increased expression of crucial melanogenic enzymes, tyrosinase and Trp1, as well as decreased Erk phosphorylation could be involved in the underlying mechanism of LfB17-34-induced melanogenesis. Further investigation of LfB17-34 will provide great opportunities to develop drugs for treating hypopigmentation disorders, such as graying of hair and vitiligo in the future.

\section{Acknowledgements}

This study was supported by the grant from National Taiwan University (no. 105A118).

\section{References}

1. Tobin DJ: The cell biology of human hair follicle pigmentation. Pigment Cell Melanoma Res 24: 75-88, 2011.
2. Dessinioti C, Stratigos AJ, Rigopoulos D and Katsambas AD: A review of genetic disorders of hypopigmentation: Lessons learned from the biology of melanocytes. Exp Dermatol 18: 741-749, 2009.

3. Panhard S, Lozano I and Loussouarn G: Greying of the human hair: A worldwide survey, revisiting the '50' rule of thumb. Br J Dermatol 167: 865-873, 2012.

4. Ezzedine K, Eleftheriadou V, Whitton $\mathrm{M}$ and van Geel N: Vitiligo. Lancet 386: 74-84, 2015.

5. Falabella R and Barona MI: Update on skin repigmentation therapies in vitiligo. Pigment Cell Melanoma Res 22: 42-65, 2009.

6. Hsiao JJ and Fisher DE: The roles of microphthalmia-associated transcription factor and pigmentation in melanoma. Arch Biochem Biophys 563: 28-34, 2014.

7. Gifford JL, Hunter HN and Vogel HJ: Lactoferricin: A lactoferrin-derived peptide with antimicrobial, antiviral, antitumor and immunological properties. Cell Mol Life Sci 62: 2588-2598, 2005.

8. Centeno JM, Burguete MC, Castelló-Ruiz M, Enrique M, Vallés S, Salom JB, Torregrosa G, Marcos JF, Alborch E and Manzanares P: Lactoferricin-related peptides with inhibitory effects on ACE-dependent vasoconstriction. J Agric Food Chem 54: 5323-5329, 2006.

9. Fernández-Musoles R, López-Díez JJ, Torregrosa G, Vallés S, Alborch E, Manzanares P and Salom JB: Lactoferricin B-derived peptides with inhibitory effects on ECE-dependent vasoconstriction. Peptides 31: 1926-1933, 2010.

10. Ruiz-Giménez P, Ibáñez A, Salom JB, Marcos JF, López-Díez JJ, Vallés S, Torregrosa G, Alborch E and Manzanares P: Antihypertensive properties of lactoferricin B-derived peptides. J Agric Food Chem 58: 6721-6727, 2010.

11. Yan D, Chen D, Shen J, Xiao G, van Wijnen AJ and Im HJ: Bovine lactoferricin is anti-inflammatory and anti-catabolic in human articular cartilage and synovium. J Cell Physiol 228: 447-456, 2013.

12. Nakajima M, Shinoda I, Samejima Y, Miyauchi H, Fukuwatari Y and Hayasawa $\mathrm{H}$ : A simple and quantitative cell-blotting assay for evaluation of pigmentation of cultured melanocytes and its use in demonstrating the depigmenting effect of lactoferrin. Arch Dermatol Res 289: 180-183, 1997.

13. Hsu WM, Che MI, Liao YF, Chang HH, Chen CH, Huang YM, Jeng YM, Huang J, Quon MJ, Lee H, et al: B4GALNT3 expression predicts a favorable prognosis and suppresses cell migration and invasion via $\beta_{1}$ integrin signaling in neuroblastoma. Am J Pathol 179: 1394-1404, 2011.

14. Lee J, Jung E, Park J, Jung K, Park E, Kim J, Hong S, Park J, Park S, Lee S, et al: Glycyrrhizin induces melanogenesis by elevating a cAMP level in b16 melanoma cells. J Invest Dermatol 124: 405-411, 2005.

15. Bellei B, Maresca V, Flori E, Pitisci A, Larue L and Picardo M: p38 regulates pigmentation via proteasomal degradation of tyrosinase. J Biol Chem 285: 7288-7299, 2010.

16. Wang D, Xu X, Ma H, Yue X, Li C and Zhu W: Optimization of the method for the culture of melanocyte precursors from hair follicles and their activation by 1,25-dihydroxyvitamin D3. Exp Ther Med 6: 967-972, 2013.

17. Che MI, Huang J, Hung JS, Lin YC, Huang MJ, Lai HS, Hsu WM, Liang JT and Huang MC: $\beta 1,4-N$-acetylgalactosaminyltrans ferase III modulates cancer stemness through EGFR signaling pathway in colon cancer cells. Oncotarget 5: 3673-3684, 2014.

18. Wu M, Hemesath TJ, Takemoto CM, Horstmann MA, Wells AG, Price ER, Fisher DZ and Fisher DE: c-Kit triggers dual phosphorylations, which couple activation and degradation of the essential melanocyte factor Mi. Genes Dev 14: 301-312, 2000.

19. Bae JS, Han M, Yao C and Chung JH: Chaetocin inhibits IBMX-induced melanogenesis in B16F10 mouse melanoma cells through activation of ERK. Chem Biol Interact 245: 66-71, 2016.

20. Son YO, Lee SA, Kim SS, Jang YS, Chun JC and Lee JC: Acteoside inhibits melanogenesis in B16F10 cells through ERK activation and tyrosinase down-regulation. J Pharm Pharmacol 63: 1309-1319, 2011. 